
internationales

vol. $19-n^{\circ} 3 \mid 2003$

Moyen-Orient : mutations récentes d'un carrefour migratoire

\title{
L'impact du capital social sur le processus de rapatriement des réfugiés palestiniens
}

Une étude préliminaire des réseaux économiques et sociaux de parenté en Palestine et en Israël

\section{Sari Hanafi}

\section{(2) OpenEdition}

\section{Journals}

Édition électronique

URL : https://journals.openedition.org/remi/468

DOI : $10.4000 /$ remi.468

ISSN : $1777-5418$

Éditeur

Université de Poitiers

Édition imprimée

Date de publication : 12 décembre 2003

Pagination : 43-72

ISBN : 2-911627-35-0

ISSN : 0765-0752

Référence électronique

Sari Hanafi, «L'impact du capital social sur le processus de rapatriement des réfugiés palestiniens », Revue européenne des migrations internationales [En ligne], vol. $19-n^{\circ} 3$ | 2003, mis en ligne le 09 juin 2006, consulté le 15 avril 2022. URL : http://journals.openedition.org/remi/468 ; DOI : https://doi.org/ $10.4000 /$ remi.468

Ce document a été généré automatiquement le 15 avril 2022.

(c) Université de Poitiers 


\section{L'impact du capital social sur le processus de rapatriement des réfugiés palestiniens}

Une étude préliminaire des réseaux économiques et sociaux de parenté en Palestine et en Israël

\section{Sari Hanafi}

1 Les migrations internationales sont souvent étudiées du point de vue de la théorie économique du push and pull. Certains chercheurs ont néanmoins commencé à reconnaître la complexité de ce phénomène et à le situer dans son contexte social, culturel, politique et économique. Actuellement trois processus sont pertinents pour expliquer la migration internationale : le premier concerne la circulation intense du capital, des marchandises et services (la globalisation économique); le deuxième concerne les systèmes politiques centralisés qui opèrent en parallèle tout en créant (paradoxalement) des frontières imperméables et en empêchant la mobilité individuelle, tandis que le troisième processus est à mettre en relation avec le caractère virtuel de certains mouvements globaux qui se manifestent à travers les nouveaux moyens de communication et les nouvelles technologies médiatiques: Internet et courrier électronique. Ces processus ont intensifié la création de nouveaux réseaux sociaux et économiques et ont modifié les modèles d'immigration et de migration de retour.

2 La Guerre du Golfe, la conférence de paix à Madrid en 1991 et les Accords d'Oslo en 1993, représentent autant de ruptures dans l'évolution de la situation au Moyen-Orient. Dans le contexte palestinien, le "droit au retour» des réfugiés, soit dans leur lieu d'origine (avant 1948) soit vers une entité politique palestinienne, a été l'une des questions les plus disputées dans les négociations avec le gouvernement israélien au cours de la dernière décennie.

3 L'expérience du rapatriement de réfugiés dans plusieurs pays montre que les aides des gouvernements ou des organisations internationales sont limitées dans ces opérations. Le capital social semble jouer un rôle important pour soutenir les réfugiés, surtout au 
début du processus ${ }^{1}$. Dans ce texte, nous examinons le capital social dont les éventuels rapatriés palestiniens pourraient bénéficier, s'il leur était permis de revenir. Cette question sera examinée à travers une analyse des réseaux transnationaux de parenté dans leurs aspects sociaux et économiques. Notre démarche s'appuie principalement sur une enquête socioéconomique et anthropologique réalisée par questionnaire ouvert. Elle met l'accent sur les divers types de réseau auxquels les individus ont accès, et accorde une attention particulière à la compréhension des processus par lesquels se fait l'inclusion ou l'exclusion par rapport à ces réseaux. Elle touche également à des questions plus subjectives telles que celles qui concernent les perceptions de la confiance et de la réciprocité normative ${ }^{2}$.

4 Cet article cherche à identifier les modèles de retour et de transnationalité. Il met en relief les liens de parenté sociale et économique entre les Palestiniens à l'intérieur et à l'extérieur des Territoires Palestiniens, ainsi que le mode d'entreprenariat qui s'y développe. À la différence d'autres études qui portent sur l'absorption de réfugiés, centrées uniquement sur la contribution de l'État et de la communauté internationale pour faciliter le retour, nous nous intéressons à l'examen du capital social, qui peut encourager le retour ou l'adaptation au nouvel environnement. Ce texte sera centré sur les facteurs objectifs qui influencent la décision du retour, tels que les réseaux sociaux et économiques de la parenté, l'entreprenariat et la culture migratoire, et non sur les attitudes fondées sur les émotions, qui expriment la position politique du sujet mais pas nécessairement son intention concernant le retour ${ }^{3}$. Bien que nous ayons pris en compte certaines attitudes émotionnelles, dans ce texte, nous ne leur avons pas attribué un poids important, ceci à cause de la faiblesse de leur pouvoir analytique.

Quel type de retour aura lieu? Quel sera le profil des rapatriés (returnees) ? Y aura-t-il des masses de réfugiés qui se précipiteront en même temps, ou plutôt des filets de groupes fragmentés ? Quelle sera la motivation du retour : le nationalisme et le désir de stabiliser l'identité (ou les identités) après l'expérience de l'exil, ou plutôt quelque chose qui les dépasse ? Dans le cas où Israël accepte le principe du droit au retour, ce dernier sera-t-il volontaire ou forcé ? Qu'est-ce qui constitue, au fait, le retour « dans la sécurité et la dignité » ? Les réfugiés devraient-ils être obligés de "revenir » ailleurs dans le pays s'ils ne peuvent pas retourner dans leurs localités d'origine? Voilà plusieurs questions auxquelles cette étude vise à répondre.

6 Cette étude adopte deux approches. Premièrement, celle du développement (development approach) qui ne concerne que les rapatriés qui choisissent de retourner chez eux (ou dans l'entité politique palestinienne) en se finançant eux-mêmes ou avec l'aide de la famille élargie, mais avec une faible intervention de l'État. Cette catégorie inclut les réfugiés palestiniens qui le feraient dans le cadre de la réunification familiale, les individus déplacés dont l'intégration est facilitée par leur profil socio-économique, et enfin, ceux qui peuvent être considérés comme des migrants économiques.

7 La deuxième approche, que nous nommons l'approche démocratique (democratic approach), aborde la population réfugiée dans son ensemble. Il s'agit alors d'étudier les réfugiés qui trouvent chez eux un plan d'intégration économique et sociale, qu'ils proviennent des camps, des grandes villes, ou de pays riches comme les Monarchies du Golfe. Pour ces deux approches, le retour inclut les réfugiés reconnus mais de statut fragile car ne disposant que d'un document de voyage, les réfugiés venus de la diaspora et munis d'un passeport d'un pays hôte, ainsi que les migrants économiques. 
8 L'examen de la migration de retour dans la perspective de l'analyse des réseaux sera fructueux pour les objectifs de notre analyse. Nous nous appuierons sur des études qui prennent en compte une large gamme de facteurs qui influent sur les possibilités et le potentiel du retour, y compris le rôle des réseaux de parenté, les modèles de dispersion des réfugiés, et les profils socioéconomiques avant et après le retour. Entre autres facteurs, nous considérerons le désir de réfugiés de retourner dans leur patrie - ou du moins dans une patrie - et la réalisation probable du projet de retour, que ce dernier soit immédiat ou différé.

Hypothèses de la recherche

9 Pour atteindre les objectifs de cette étude nous proposons quatre hypothèses.

10 Le premier est de considérer que l'action économique est imbriquée dans la structure sociale. Aussi importe-t'il d'étudier les réseaux sociaux. Qu'est-ce que cette imbrication? Comment comprendre le comportement économique des rapatriés en relation avec celui des entrepreneurs locaux, cela en rapport avec le mouvement de retour et plus généralement, l'intensification de la mobilité transnationale?

11 Pour comprendre le comportement économique des entrepreneurs palestiniens, il faut prendre en compte la nouvelles littérature en sociologie économique, notamment le concept d'« imbrication » (embeddedness) de Polanyi et de Granovetter (Polanyi, 1957; Granovetter and Swedberg, 1995). Ce concept se réfère au fait que divers types de transactions économiques sont insérés dans des structures sociales et politiques englobantes qui affectent leurs conséquences. Donc, on ne peut pas comprendre les transactions économiques des Palestiniens sans se référer au statut social et juridique des communautés auxquelles ils appartiennent.

12 À cet égard, l'homme ou la femme d'affaires palestiniens n'est pas un homo economicus qui cherche à maximiser le profit tout en agrandissant le marché pour son pays, mais plutôt un "acteur humain » qui cherche à satisfaire ses besoins en jouant un rôle en tant que membre d'un réseau ethnique. Néanmoins, l'acteur humain n'est pas un être abstrait, purement patriotique, qui cherche à « envoyer » du capital vers sa patrie. Il est avant tout un individu qui calcule son investissement économique de manière complexe, qui prend en compte l'avenir plus que le présent en termes économiques, et qui cherche le prestige social et le pouvoir politique, ce dernier au sens étroit et aussi au sens plus large de Foucault.

13 La deuxième hypothèse concerne la place des liens familiaux par rapport aux objectifs de notre étude. Comme nous l'avons déjà mentionné, nous essayons d'identifier les divers niveaux des structures où l'individu, sujet de la migration de retour, s'insère (amitié, famille élargie, liens villageois et nationaux). Néanmoins, pour ce qui concerne la décision de migrer et le soutien dont pourront disposer les réfugiés palestiniens pour leur absorption, la famille nucléaire et élargie demeure, à notre avis, l'acteur principal. Un des objectifs du terrain a donc été d'identifier les divers types de liens familiaux : forts, faibles, ou détruits.

14 La troisième hypothèse concerne un modèle de rapatriement qui n'est pas seulement définitif mais aussi transnational, ainsi qu'on le constate dans de nombreuses études de réfugiés dans le monde. Ceci veut dire que les réfugiés/rapatriés entretiendraient des liens avec la vie politique, sociale et économique, aussi bien dans la patrie dans laquelle ils retournent que dans leur pays de résidence précédent. 
La dernière hypothèse de cette étude est de caractère politique. Le projet de deux États-nations est toujours populaire auprès de la majorité de Palestiniens tout comme des Israéliens. Ce qui implique l'établissement d'un système d'apartheid où la question de l'hégémonie des Juifs dans l'État d'Israël ne sera pas mise en cause dans un avenir proche. L'enjeu concerne alors le type d'État-nation que les Israéliens et, de leur côté, les Palestiniens vont construire. Dans le cas où Israël reconnaîtrait le droit au retour des Palestiniens, leur rapatriement sera sujet de considérations sociologiques et économiques. Dans le cas contraire, le quota et le profil des réfugiés influeront beaucoup sur les choix des Palestiniens à l'égard de leur future mobilité.

Les facteurs influençant les mouvements de réfugiés

Plusieurs facteurs influencent la décision du réfugié de rentrer dans son pays d'origine ou de choisir une autre option. Nous nous pencherons sur certains éléments d'ordre socio-économique qui touchent les réfugiés palestiniens (et plus généralement, les Palestiniens qui vivent à l'étranger), dans le pays hôte et dans l'espace de retour (les Territoires Palestiniens ou Israël). Ce ne sont cependant pas les seuls éléments importants ; par exemple, le facteur géographique, la distribution des populations dans l'espace de l'État d'Israël, est pertinent dans la prise de décision du réfugié. Le travail d'Abu-Sitta (2001) montre que les zones autour des anciens villages palestiniens demeurent vides et inutilisées, et qu'elles seraient capables d'absorber des réfugiés rapatriés sans difficulté. Pour l'auteur, cette aire rurale vide correspond à l'héritage paysan de la majorité des réfugiés palestiniens. Néanmoins, on doit se demander si, après 55 ans d'absence, ces réfugiés et leurs descendants, qui habitent majoritairement des métropoles telles que Damas, Amman, Le Caire, sans parler de Chicago et de New York, pourraient redevenir des paysans. Par ailleurs, selon l'enquête PSR de 2003 dans les Territoires Palestiniens, les maisons d'environ la moitié des originaires des zones qui se trouvaient dans la Palestine de 1948 ont été détruites; or $40 \%$ des enquêtés affirment qu'ils ne retourneront pas dans leur espace d'origine dès lors que leur maison familiale n'existe plus.

Mais la capacité d'absorber les réfugiés sur le plan spatial n'est pas le facteur déterminant dans les scénarios de retour. Les Irlando-Américains ne sont pas retournés en Irlande après la fin du colonialisme britannique. Peu d'Arméniens sont retournés en Arménie après l'indépendance de ce pays, et peu de Libanais ont rejoint leur pays d'origine après la fin de la guerre civile. Pourtant, dans tous ces cas, il y avait non seulement une capacité largement suffisante, mais aussi une volonté politique de réabsorption.

8 Un autre facteur à prendre en compte, mais qui dépasse l'optique de notre étude, concerne le système d'assistance sociale dans les pays hôtes comparé avec celui des zones de rapatriement. Par exemple, en comparaison avec Israël, les services de santé, d'assistance sociale et d'éducation dans les Territoires palestiniens et en Jordanie sont peu développés. Ce facteur positif encouragera-t-il les Palestiniens à retourner en Israël plutôt que dans les Territoires palestiniens ? ${ }^{4}$

Finalement, la possibilité de retour des réfugiés palestiniens est étroitement liée à trois éléments : d'abord le droit au retour, ensuite la situation urbaine des camps de réfugiés, et enfin la position des pays hôtes arabes. Les trois scénarios élaborés dans la conclusion sont conditionnés par ces éléments et surtout par la mesure dans laquelle le droit au retour sera effectif. Il importe de souligner que de nombreuses études font état du peu d'espoir quant à une solution politique de la situation. Une enquête du PSR en 
2003 montre qu'environ la moitié de ceux qui ont répondu accepte l'idée de remettre indéfiniment la question des réfugiés, jusqu'à l'établissement d'un État palestinien. À cet égard, l'étude de Najeh Jarrar (2003), où se combinent des méthodologies sociologiques et anthropologiques, est révélatrice. Tandis que les réfugiés montrent dès 1994, la date de l'enquête de Jarrar, un manque de confiance dans le processus de paix, on constate qu'ils sont prêts à chercher une solution réaliste à leur situation ${ }^{5}$. Ce constat apparaît clairement dans les entretiens approfondis, où environ le quart des répondants se dit prêt à accepter une compensation et presque la moitié à rentrer sous gouvernement israélien (tableau 1).

Tableau 1 : Attitudes des réfugiés concernant les solutions réalistes, 1993

\begin{tabular}{|l|c|c|c|c|c|c|}
\hline Camp & \multicolumn{2}{|c|}{ Rapatriement sous gouvernement israélien } & \multicolumn{3}{c|}{ Compensation } \\
\hline & D'accord & Ne sais pas & Pas d'accord & D'accord & Ne sais pas & Pas d'accord \\
\hline Fari'a & 41,7 & 6,3 & 52,0 & 29,8 & 4,3 & 65,9 \\
\hline Balata & 54,8 & 4,1 & 41,1 & 14,2 & 6,8 & 74,0 \\
\hline Moyenne & 49,6 & 5,0 & 45,4 & 23,9 & 5,8 & 70,8 \\
\hline
\end{tabular}

20 Parmi ces facteurs, nous analyserons la densité des liens transnationaux de parenté et le rôle que pourra jouer la culture de migration des Palestiniens. Nous nous interrogerons aussi sur l'influence de l'expérience des retours d'exilés dans les territoires palestiniens ces dernières années, ainsi qu'aux conséquences que pourraient avoir les conditions de vie dans les camps palestiniens sur leur plus ou moins grande propension au retour dans les années à venir.

La densité des liens transnationaux de parenté

21 Nous nous intéresserons ici à une seule forme de capital social, celle des liens sociaux. Le réseau familial élargi est un filet de sécurité important pour les migrants. Néanmoins, nous nous demanderons quels sont les effets des ruptures aggravées par l'occupation et par les frontières nationales sur les réseaux transnationaux de parenté des Palestiniens. Nous nous poserons plusieurs questions sur les aspects sociologiques des réseaux palestiniens : comment mesurer la force d'un lien? Les relations de proximité relient-elles des individus semblables? Les liens entre gens dissemblables sont-ils plus faibles?

Nous sommes conscient du fait que certains types de capital social représentent des obstacles plutôt que des appuis pour le migrant de retour, par exemple quand les normes du groupe obligent les membres à partager plutôt qu'à accumuler les richesses, ou quand elles bloquent l'accès aux services (Portes, 1998).

La connectivité entre les membres du réseau se fonde sur une gamme de choix faits par les individus et les membres de leurs familles. Mesurer le degré de connexion entre eux est une question subjective ; cependant l'enquête Shaml cerne les facteurs structurels qui peuvent conditionner et limiter les choix des migrants. Les difficultés de la traversée des frontières, la fréquence des visites, des appels téléphoniques et de l'utilisation du courrier électronique représentent des indicateurs de la connectivité du réseau familial. Tous ces facteurs conditionnent les liens entre les membres de la famille et influencent ainsi la décision de retour.

Des familles transnationales

Dans les Territoires palestiniens, on constate un niveau de transnationalité élevé entre parents proches, puisque $40 \%$ de ces parents vivent au-delà de la frontière. Un peu plus 
du quart d'entre eux peut rendre visite à sa famille en Palestine ou en Israël. Par contre, les familles des personnes interviewées en Israël sont beaucoup moins dispersées, à peine $15 \%$ de leurs membres vivent dans un autre pays (tableau 2).

Tableau 2 : Membres des familles des interviewés : résidence à l'étranger, possibilité de visites et statut ou non de réfugié

\begin{tabular}{|c|c|c|c|c|}
\hline \multicolumn{5}{|c|}{ PARENTS PROCHES : PÈRE, MÈRE, CONJOINTS, ENFANTS } \\
\hline & \multicolumn{4}{|c|}{ Entretiens réalisés dans les Territoires palestiniens } \\
\hline & Total & Résidant à l'étranger & \begin{tabular}{|l|} 
Pouvant rendre visite \\
en Palestine ou Israzl
\end{tabular} & Réfugiés \\
\hline $\begin{array}{l}\text { Nombre moyen de } \\
\text { parents }\end{array}$ & 12,1 & 4,86 & 3,42 & 5,92 \\
\hline $\begin{array}{l}\text { Pourcentage selon } \\
\text { certains critères }\end{array}$ & 100 & 40 & 28 & 49 \\
\hline \multicolumn{5}{|c|}{ Entretiens réalisés en Isräl } \\
\hline $\begin{array}{l}\text { Nombre moyen } \\
\text { de parents }\end{array}$ & 9,69 & 1,48 & 1,20 & 2,12 \\
\hline $\begin{array}{l}\text { Pourcentage selon } \\
\text { certains critères }\end{array}$ & 100 & 15 & 12 & 22 \\
\hline \multicolumn{5}{|c|}{$\begin{array}{c}\text { PARENTS AU } 2^{\circ} \text { DEGRÉ (ONCLES ET TANTES MATERNELS } \\
\text { OU PATERNELS ET LEUR FAMILLE) }\end{array}$} \\
\hline $\begin{array}{l}\text { Nombre moyen } \\
\text { de parents }\end{array}$ & 6,52 & 4,95 & 3,24 & 5,13 \\
\hline $\begin{array}{l}\text { Pourcentage répondant } \\
\text { à certains critères }\end{array}$ & 100 & 76 & 50 & 79 \\
\hline
\end{tabular}

Le pourcentage de ceux qui se trouvent à l'étranger est beaucoup plus important pour les parents au deuxième degré $(76 \%)$ que pour les parents proches. Seulement la moitié d'entre eux ont la possibilité de rendre visite à leur famille en Palestine ou en Israël. Plus de $79 \%$ de ces parents sont des réfugiés. Dans cette catégorie, les liens sociaux et économiques sont beaucoup moins fréquents ; parmi les répondants dans les Territoires palestiniens seuls $15 \%$ reçoivent une aide de ces parents vivant hors du pays, et ils sont encore moins nombreux (8\%) parmi ceux qui ont été interrogés en Israël. Par ailleurs, on observe très peu de projets économiques entre cette catégorie de Palestiniens à l'étranger et les répondants. $6 \%$ des Palestiniens dans les Territoires reçoivent de l'aide des parents dans cette catégorie au début de leur vie professionnelle.

Le rapport à d'autres parents, moins proches que les deux catégories décrites ci-dessus, est très faible. Un tiers des répondants déclare n'avoir aucun lien avec eux et seulement 25 \% déclarent avoir certains liens. La grande majorité affirme n'avoir avec ce cercle plus lointain de leur parenté aucune relation économique.

En bref, le degré de la transnationalité suggère un mode de migration de retour plutôt transnational que de retour définitif en bénéficiant de réseaux parentaux.

La connectivité : une construction géographique et dépendant du genre

Le lieu de naissance des interviewés joue un rôle important dans leur connexion avec les membres de leur famille qui y habitent toujours. Par exemple, parmi ceux qui sont nés en Jordanie, $79 \%$ ont maintenu des liens avec leurs proches parents dans ce pays ${ }^{7}$. Néanmoins, ce lien s'effrite avec le temps pour les rapatriés, ceux qui sont revenus 
s'installer dans les Territoires palestiniens. La quasi impossibilité de traverser la frontière explique cette situation.

Nous avons demandé aux réfugiés interviewés ce qu'ils envisageaient de faire de leurs propriétés immobilières dans le pays hôte après leur retour. La grande majorité affirme qu'ils les garderaient et les loueraient : les réfugiés ne veulent pas couper les liens avec le pays qui les a accueillis.

30 L'hypothèse que les liens les plus proches sont avec les membres de la famille plutôt qu'avec des amis est confirmée par le fait que, lorsqu'on les interroge sur leurs relations, seulement $5 \%$ des interviewés mentionnent seulement les amis et $8 \%$ associent la famille et les amis dans leurs réponses. Les hommes affirment plus souvent que les femmes que leurs liens sociaux les plus étroits sont ceux qui les unissent aux membres de leur famille. On remarque aussi des différences liées à l'âge : les jeunes évaluent leurs rapports avec les frères et sœurs au-dessus de ceux avec leurs cousins et amis. Par contre, les rapatriés donnent plus d'importance aux relations avec les amis.

31 Les hommes palestiniens que nous avons interviewés en Palestine ou en Israël ont perdu le contact avec le tiers de leurs parents proches et rencontrent les autres tous les deux ans environ, tandis que les femmes ont moins perdu le contact ( $29 \%$ pour les interviewées dans les Territoires, et $14 \%$ pour celles qui vivent en Israël). En général, les hommes ont moins de contacts avec leur parenté que les femmes. Par contre, ils ont avec leurs sœurs et leurs filles des contacts plus fréquents qu'elles. De même, les hommes qui vivent dans les Territoires palestiniens appellent plus souvent leurs sœurs que leurs frères au téléphone.

32 L'enquête fait aussi état du peu de contact entre les hommes d'une part, leur bellefamille et leurs parents par alliance d'autre part. La proximité au niveau de l'âge joue un rôle important par rapport aux connexions sociales, mais moins en ce qui concerne les liens économiques. On peut expliquer ce constat par le décalage générationnel qui se manifeste dans les contextes palestiniens et arabes, surtout en rapport avec la transnationalité. La deuxième Intifada limite la possibilité de contacts entre les Palestiniens dans les Territoires occupés et entre ces derniers et leurs proches à l'étranger. La situation est particulièrement grave pour ceux qui habitent Gaza, où l'armée israélienne a imposé la fermeture totale de la bande.

La localité où habitent les interviewés est très pertinente quant aux contacts avec la parenté. Dans la bande de Gaza, $43 \%$ des répondants ne rencontrent jamais leurs parents proches, ne leur rendant ou ne recevant pas leur visite. Ce pourcentage est plus élevé chez ceux qui vivent dans les camps, puisque les parents de ces derniers sont plus souvent exilés. Les Palestiniens qui habitent les grandes villes israéliennes comme Haïfa par exemple ont perdu les contacts directs avec leurs parents proches. Les visites diminuent avec le vieillissement, quelle que soit la classe sociale, bien qu'elles restent plus fréquentes chez les mieux scolarisés. Il apparaît que l'éducation entraîne la possibilité de voyages et de rencontres avec des proches établis à l'étranger. L'utilisation du téléphone ne semble pas compenser l'absence physique; il y a peu d'appels entre les gens qui ne se rencontrent pas. De façon générale, la majorité des répondants téléphone à sa famille tous les deux mois environ. Ceux qui conservent une propriété en Palestine rencontrent plus souvent leur famille.

34 La frontière représente un obstacle majeur aux rencontres : ainsi $56 \%$ de ceux qui sont interdits de séjour en Palestine/Israël ont perdu le contact avec leurs parents proches. La distribution spatiale joue aussi un rôle ; les réfugiés palestiniens en Jordanie et en 
Égypte maintiennent le contact avec la famille plus souvent que ceux qui sont installés dans les États du Golfe, mais même ces derniers la voient environ tous les deux ans. Par contre, les rencontres familiales avec les parents vivant au Liban ou en Irak sont presque inexistantes. En fait, les Palestiniens établis en Europe et aux États-Unis peuvent rencontrer leur parenté en Palestine ou en Israël plus souvent que ceux qui vivent dans les pays arabes, ceci probablement à cause de la difficulté, sinon de l'impossibilité, des voyages entre ces deux pays.

La plupart des appels téléphoniques concerne les enfants adultes installés à l'étranger, au rythme d'environ un tous les deux mois, sinon plus souvent. Les appels sont très importants pour les répondants qui habitent à Bethlehem, Walaja (banlieue de Jérusalem), Ramallah et les camps, moins pour ceux qui vivent à Naplouse et dans la bande de Gaza. Les appels d'Israël vers l'étranger sont assez rares, sauf pour Um al Fahm, où ils représentent un geste de solidarité, semble-t-il, par rapport aux parents en Cisjordanie et en Jordanie. La fréquence des contact avec la famille établie à l'étranger est la même pour les hommes et pour les femmes qui habitent les Territoires palestiniens, mais les femmes en Israël se servent du téléphone moins souvent que les hommes. Ceci est le cas également pour les moins scolarisés. Les appels sont plus fréquents vers la famille proche qui se trouve en Europe et aux États-Unis qu'avec la parenté résidant dans le Golfe, en Égypte ou en Jordanie, probablement à cause du coût moins élevé des communications pour les deux premières destinations. Le contact avec le Liban est rare. Une répondante dit donner de l'argent pour douze cartes téléphoniques à un oncle vieux et malade en Jordanie, pour qu'il puisse appeler sa famille en Palestine.

L'utilisation du courrier électronique entre les membres de la famille immédiate qu'ils vivent en Palestine ou en Israël est peu fréquent; il concerne seulement le tiers des effectifs, et la plupart des utilisateurs ont moins que 40 ans. Les femmes le pratiquent moins souvent que les hommes en Palestine, mais la situation est inverse en Israël. Le contact par Internet prend plusieurs formes, y compris les "chats", le SMS et l'échange de photos.

Le mariage endogame représente un indicateur de la connectivité des réseaux familiaux. De nombreuses études témoignent de la persistance d'un taux d'endogamie élevé chez les Palestiniens. Selon Pedersen et al. (2001, pp. 80-84), "Dans la société palestinienne, la famille, et en particulier les parents, joue un rôle important dans la sélection d'un conjoint qui convienne ». Seules $36,6 \%$ des femmes nées entre 1940 et 1949 et $43 \%$ de celles qui sont nées entre 1960 et 1969 se marient en dehors du groupe de parenté ou hamula. Les mariages sont souvent arrangés, comme c'est le cas dans la plupart des pays du monde arabe. La mère joue un rôle informel dans le processus, tandis que le père est celui qui prend la décision finale (Tuastad 1993, 1996). L'endogamie est un trait prééminent du mariage arabe - le mariage au sein du même lignage, secte, communauté, groupe, village ou quartier. Les unions entre les cousins germains et entre les consanguins sont les formes les plus courantes de l'endogamie dans cette partie du monde (Holy 1989).

L'enquête Shaml tout comme les travaux de Riina Isotalo $(1997,2002)$ démontre que la pratique de l'endogamie persiste même au niveau transnational, bien qu'à un moindre degré. Ceci s'explique par le fait que les mariages endogames transnationaux sont très dispendieux, tandis que les mariages endogames locaux sont avantageux sur le plan économique ${ }^{8}$. 
Solidarité et liens économiques

Le capital social se réfère à la capacité de l'individu à mobiliser des ressources rares à travers son appartenance à des réseaux et des regroupements sociaux plus larges. Ces ressources concernent des faveurs économiques comme la réduction des prix, le crédit sans intérêt, l'information sur les conditions économiques, etc.

Nous distinguons deux formes d'assistance entre parents. La première concerne l'aide unidirectionnelle et la deuxième, l'entraide réciproque. Selon la théorie des échanges, le réseau est beaucoup plus fort quand l'assistance n'est pas réciproque, puisque « dans le décalage entre le don et le retour, l'obligation, la confiance et la coopération se créent entre les partenaires » (Uehara 1990 : 524, cité par Jaconsen et Deeb, 2003 : 223).

La solidarité entre les Palestiniens des Territoires et les exilés est assez importante. Un tiers des répondants affirme recevoir de l'aide et cela dès avant l'actuelle Intifada; autrement dit, cette aide n'est pas le fait des difficultés économiques accrues depuis'. Cette assistance, financière et autre, concerne surtout les parents et les enfants, les frères et sœurs. Nous étendons le concept de l'aide pour y inclure les cadeaux et l'aide fournie pour couvrir les coûts de l'éducation ou des traitements médicaux. L'aide pour l'éducation ne concerne que les parents masculins. Les fêtes comme l'Aïd (fête musulmane) et Noël sont également des occasions pour des cadeaux.

On constate avec intérêt qu'environ la moitié de l'aide est transférée à travers les banques, changement marqué par rapport aux années soixante-dix et quatre-vingt, époque où, selon plusieurs études, les échanges se faisaient par la voie personnelle. Cette aide est devenue rare pour les Palestiniens en Israël, probablement à cause de leur situation économique relativement favorable ainsi que de leurs liens transnationaux plus faibles.

3 Pour ce qui concerne l'entraide réciproque, on en trouve peu de cas au niveau transnational. Fait curieux, l'aide ne va pas seulement vers les Territoires palestiniens mais aussi ailleurs; un tiers des répondants envoie de l'aide à partir des Territoires palestiniens vers leurs parents installés à l'étranger. Ce type de relation coutumière est indépendant de la situation économique; ainsi bien des parents envoient une aide financière à leurs filles, même quand ces dernières se trouvent à l'étranger.

44 Tandis que la solidarité entre les Territoires palestiniens et l'étranger est importante, le partenariat économique est très difficile, compte tenu de la situation actuelle : faire venir des partenaires de la diaspora parait hasardeux, bien qu'une certaine aide soit fournie qui permet aux petits commerçants de monter leur entreprise.

Il apparaît que l'aide, en Palestine comme en Israël répond à des motifs altruistes et non pas matérialistes, puisqu'elle implique un transfert de ressources motivé par des normes ou par une solidarité avec les besoins et les objectifs du groupe d'appartenance, plutôt qu'une réciprocité ou une confiance obligatoires qui seraient basées sur une attente de prestige dans la communauté et de services en retour imposés par des sanctions collectives (Portes, 1985). Cette situation sera un atout important pour la réinsertion des exilés rapatriés, surtout dans sa phase initiale.

Le contact en face à face est très important dans les transactions économiques, surtout lorsqu'il est question d'entraide. Une étude du Fafo (Sletten et Pedersen, 2003: 47) montre que les habitants des Territoires comptent sur leurs parents à l'étranger, bien que l'impossibilité de toute communication directe dresse un obstacle pour beaucoup 
de transactions. Selon l'étude Shaml, à peu près la moitié des transferts ne passe pas par les banques mais l'argent est apporté à cadeau lors des visites familiales.

La famille nucléaire fracturée

47 Des recherches de terrain récentes montrent un tableau de réseaux fracturés à l'intérieur de la famille nucléaire dans les Territoires palestiniens, à la différence d'Israël. (Hanafi, 2001; Isotalo, 2002). Les mères et les enfants habitent dans un pays, tandis que les pères et maris vivent et travaillent dans un autre, et les grands parents et d'autres parents demeurent dans un autre encore (voir les études effectuées par l'organisation israélienne pour les droits de l'Homme, B'tselem $\left.{ }^{10}\right)$. Cette fragmentation familiale suggère que les retours ne réunifieront pas toute la famille, surtout dans les premières années.

Généralement ces sont les hommes qui décident des déplacements familiaux; ces décisions dépendent de plusieurs facteurs, notamment ceux du marché. Cependant, l'individu qui a trouvé un emploi (l'homme sauf exception) se fait rejoindre par sa famille et prend les décisions relatives à la mobilité du groupe. Les statistiques montrent que le pourcentage de migrants internationaux féminins s'accroît; actuellement elles représentent $47,5 \%$ du total. La décision de migrer dépend également des possibilités qui s'offrent aux enfants en matière d'éducation.

La culture de la migration

Selon la théorie du capital humain, la migration se produit si les gains escomptés des coûts de la migration sont plus importants que les gains escomptés dans le pays d'origine. Cette théorie est l'objet d'une remise en question de la part de plusieurs auteurs, et on apporte aujourd'hui une attention accrue à l'action des réseaux migratoires (Massey et al., 1993). L'enquête socio-anthropologique Shaml fournit des renseignements détaillés sur la migration et la culture de la migration, et ceci pour deux types de mouvements migratoires : d'une part la migration permanente, d'autre part la migration circulaire et temporaire à l'intérieur de la Palestine ou d'Israël et au niveau international. Pour comprendre cette dernière, l'histoire migratoire des adultes de quinze ans et plus a été recueillie, incluant la date et le lieu d'origine et de destination pour chaque mouvement enregistré.

Il n'est peut-être pas surprenant que les Palestiniens dans les Territoires soient beaucoup plus mobiles que ceux qui habitent Israël, mais moins que la population réfugiée. Souvent le mouvement est provoqué par des problèmes politiques.

51 Les Palestiniens tendent à quitter facilement de leur ville d'origine. Environ $60 \%$ des personnes interrogées ont déménagé au moins une fois, et la moyenne du nombre de déménagements est à peu près de trois. La plupart des mouvements se fait à l'intérieur des Territoires palestiniens ( $57 \%$ ), tandis que les destinations étrangères préférées sont les pays du Golfe ( $10 \%$ du total des mouvements). En Israël, les gens se déplacent moins ; seul le tiers des répondants ont déménagé trois fois ou plus, et le plus souvent ceux qui se déplacent le font dans les frontières du pays.

52 Les motifs les plus fréquents de la mobilité des habitants des Territoires palestiniens sont l'emploi, l'éducation, le mariage et la recherche d'un meilleur logement. Ce dernier motif concerne surtout les réfugiés qui veulent quitter les camps et aller s'installer en ville. Les Palestiniens en Israël présentent le mariage comme la raison la plus importante, suivie par l'emploi et l'éducation. En général le nombre des déplacements s'accroît avec le niveau de scolarité. L'analyse montre une différence selon le sexe : dans presque tous les cas, les femmes suivent les hommes ( $94 \%$ dans les 
Territoires palestiniens et $92 \%$ en Israël). Le même modèle se retrouve pour les déplacements liés à la réunification familiale: $77 \%$ des cas dans les Territoires concernent des femmes qui rejoignent des parents masculins.

Le processus de paix d'Oslo a fait diminuer le niveau de mobilité : seulement $18 \%$ des mouvements rapportés concernent la période 1993-2003. En même temps il a créé une opportunité pour des mouvements dans les Territoires palestiniens: $94 \%$ des déplacements pendant cette décennie concernent des mouvements à l'intérieur des Territoires.

L'enquête PSR de 2003 confirme l'importance de la culture de la migration et du support familial. Elle montre, entre autres, que deux tiers des habitants des Territoires palestiniens se sont déplacés au moins une fois. L'étude Fafo menée en Jordanie en 1996 était centrée sur la mobilité au cours de la vie; elle dévoile aussi le niveau élevé de mobilité de la population; la moitié des adultes s'est déplacée au moins une fois, mais pas toujours vers l'extérieur du pays (Khawaja et Tiltnes, 2002, p. 28-29).

55 L'Intifada actuelle semble avoir accentué la migration, puisque environ 100000 Palestiniens ont quitté la Cisjordanie pour la Jordanie ou pour les pays occidentaux depuis qu'elle a commencé, ce qui représente une augmentation par rapport à la période de l'occupation israélienne 1967-1993. Selon le Bureau Central de la Statistique (Israël) le taux annuel de migration était alors de 0,5 à $2 \%$ de la population, soit 5000 à 15000 personnes en Cisjordanie et 3000 à 7000 dans la bande de Gaza (CBS, 1993: 760). Il est difficile de trouver des statistiques pour la migration en cours parce que les gens ont honte de partir pendant la lutte nationale que représente l'Intifada. Pourtant un rapport de la Banque Mondiale (2003: 35$)$ se réfère à une enquête qui constate que « $2 \%$ des répondants affirment que des membres de leur famille sont partis pendant de longues périodes. " Nous pensons que les émigrants viennent des couches bien scolarisées plutôt que de la classe ouvrière. Il semble que les Palestiniens soient enclins à la mobilité, que ce soit pour émigrer ou pour revenir au pays.

Nous concluons qu'il existe deux catégories de migrants potentiels vers la Palestine ou Israël : la première inclut ceux qui n'ont pas de lien de parenté au pays. Leur " retour " dépend soit des facteurs "push» qui relèvent du pays hôte, qu'ils soient d'ordre économique, politique, social ou autre (par exemple, leur statut juridique dans le pays hôte), soit des facteurs « pull » vers l'espace Palestine / Israël. La deuxième catégorie concerne ceux qui ont accès au capital social à travers des liens de parenté dans ces régions et dont la propension au retour est beaucoup plus importante. Le capital social motive, encourage et facilite le retour.

Si l'on considère le fait que beaucoup de Palestiniens de ces deux catégories ont des liens avec le Canada et les États-Unis (enquête Fafo), la possibilité de leur migration vers ces pays est aussi importante. Le choix de ces pays et éventuellement de l'Europe et de l'Australie sera favorisé non seulement par les liens de parenté mais aussi par les systèmes d'éducation qui s'y trouvent. Environ le tiers des interviewés que nous avons interrogés dans les pays du Golfe en 1990-1995 est parti au Canada et aux États-Unis à cause de ce dernier facteur.

Le retour : une expérience assez positive

58 Dans cette réflexion sur le retour des exilés avec le retour à la paix, l'expérience des rapatriements dans les Territoires palestiniens pendant la période de transition 
associée à Oslo est instructive à plusieurs égards. Jusqu'à présent le retour en Cisjordanie et dans la bande de Gaza a eu lieu pendant deux périodes distinctes.

La première a été un mouvement forcé par le déclenchement de la première Guerre du Golfe, où quelques 350000 Palestiniens de Koweït et d'autres pays du Golfe ont été obligés d'émigrer. Seuls les 37000 d'entre eux qui avaient conservé leurs droits de résidents permanents en Cisjordanie et à Gaza sont retournés dans ces territoires (Isotalo, 2001). La deuxième période a suivi le lancement du processus de paix qui a permis le retour depuis certains pays arabes, en particulier d'Égypte, de Jordanie et de Tunisie. Les Palestiniens dont le statut dans ces pays était précaire ainsi que ceux qui bénéficiaient du quota établi par Israël et l'Autorité Nationale Palestinienne (ANP) sont revenus pendant cette deuxième période ${ }^{11}$.

60 Il y a eu des retours individuels des pays de la diaspora mais peu importants. Il s'agit de migrants de catégories socio-économiques diverses, souvent des diplômés de l'université dotés des qualifications professionnelles acquises dans les pays hôtes (Zureik, 1997). Malgré l'absence d'estimations fiables quant au nombre de rapatriés qui habitent actuellement les Territoires palestiniens, on en trouve quelques indicateurs. Le recensement PCBS de 1997 estimait le nombre total de rapatriés à 267355 , soit $10,5 \%$ de la population totale (Malki et Shalabi, 2000). Des entrevues avec des représentants du Département des Réfugiés de l'OLP indiquent qu'au moins 200000 d'entre eux sont revenus depuis le début du processus de paix d'Oslo en 1993.

61 Il convient de noter que tout discours au sujet du retour des Palestiniens vers la Cisjordanie et la Bande de Gaza comporte certains paradoxes. Les migrants de « retour » dont les localités d'origine sont devenus partie d'Israël en 1948 ne sont pas retournés à leur village ou villes d'origine; en fait, le « retour» signifie une nouvelle migration. Par ailleurs, le mouvement de « retour » demeure éphémère, puisque Israël contrôle toujours l'immigration vers les Territoires palestiniens et n'accorde généralement pas la résidence à ces immigrant. Ceux-ci possèdent un passeport étranger et sont considérés comme des touristes. On leur attribue des visas de trois mois pour les déplacements aériens et souvent des visas d'un mois seulement quand ils traversent les frontières terrestres. Même quand ils ont des emplois dans les Territoires palestiniens, ils ont peu de possibilités d'obtenir un permis de travail ou de résidence, de sorte qu'ils doivent sortir et revenir constamment. Ceux qui dépassent l'échéance de leur visa risquent l'interdiction permanente de séjour en Israël, y compris dans les Territoires palestiniens.

62 La question de la localisation du retour que nous venons d'évoquer aura

un impact sur le caractère de tout État palestinien éventuel. Comme Roger Heacock (1999: 57) l'a noté, quand le retour concerne une terre « de mémoire » (la Cisjordanie et la Bande de Gaza), plutôt qu'une localité historique (d'origine), il ne s'agit pas d'un État de "rapatriés» mais plutôt un État de colons ("settlers»). Le cas de la BosnieHerzégovine montre qu'un État fondé sur l'expérience vécue (c'est-à-dire sur l'histoire) ne réussit pas nécessairement. En revanche, Israël, dont le demi-siècle d'existence se fonde sur une mémoire discontinue et une histoire reconstruite, représente un cas de construction étatique par des colons qui a indiscutablement réussi.

Le début du mouvement de retour vers les Territoires palestiniens précède le processus de paix d'Oslo et remonte à la guerre entre l'Irak et le Koweït. Pourtant, les trois quarts des répondants à l'enquête Shaml sont revenus après Oslo (soit après 1993). La moitié est revenue grâce à l'ANP, tandis que $13 \%$ bénéficiaient de la politique de réunification 
familiale. Toujours est-il que la moitié d'entre eux n'a toujours pas le droit de résidence; par ailleurs Israël n'a pas renouvelé les visas de visite dans les Territoires depuis le début de l'Intifada actuelle.

Selon les répondants, les retours se sont effectués en consultation avec la famille ( $81 \%$ des cas) ; la famille ne s'est opposée au retour que dans $10 \%$ des cas, principalement en cas de mariages à l'étranger, que le migrant soit homme ou femme. Dans un cas, la famille a accepté la décision du père, mais les femmes ont imposé de garder leur statut d'étrangères. L'opposition au retour ne vient pas toujours de l'épouse, mais surtout des enfants plus âgés.

La situation des rapatriés semble s'améliorer après le retour. L'emploi y joue un rôle, puisque environ la moitié était employée avant le retour et $80 \%$ le sont après. Les trois quarts des rapatriés sont satisfaits de leur travail, et seulement un quart estime que leur situation économique s'est dégradée. Ces pourcentages sont dus en partie à la grande instabilité causée par l'Intifada.

environ reçoit de l'aide de la famille pour les déplacements. Ce faible niveau s'explique par le fait que la majorité de ceux qui reviennent au pays ne sont pas originaires de Cisjordanie ni de la Bande de Gaza. Ils migrent vers une localité où ils n'ont pas de famille ou bien où leur famille n'a pas les moyens de les aider.

Grosso modo, environ trois quarts des rapatriés s'affirment satisfaits de leur retour, même s'ils ont connu des difficultés. Les plus âgés sont beaucoup plus satisfaits que leurs cadets. $65 \%$ ont connu des difficultés dans la phase initiale du retour, notamment au niveau social. Environ $41 \%$ qualifient le système de santé dans les Territoires palestiniens de «mauvais » et seulement $22 \%$ le jugent «bon ». Beaucoup se plaignent des universités palestiniennes, mais plus de la moitié jugent que les écoles de leurs enfants sont bonnes. Le logement constitue un problème important, puisque $68 \%$ sont toujours locataires d'appartements dix ans après leur arrivée; ils considèrent néanmoins ces nouveaux logements comme meilleurs que ceux qu'ils ont connus avant leur retour.

On remarque que $63 \%$ des rapatriés avaient des attentes quant à la «patrie » qui étaient différentes de la réalité qu'ils y ont rencontrée. "Ce n'est pas la patrie dont je rêvais, ni le peuple auquel je croyais appartenir » dit un rapatrié arrivé après 1993 grâce à la liste de l'OLP. Néanmoins, le désenchantement n'empêche pas les rapatriés d'encourager d'autres exilés à revenir vers les Territoires palestiniens $(95 \%$ des rapatriés). Autrement dit, malgré les déceptions à l'égard de la patrie, cette dernière devient un lieu commode (et pas seulement «naturel») où habiter. Elle n'est plus synonyme seulement d'Intifada et d'aliénation politique, mais elle devient un pays "d'intérêt et d'amour », aux dires d'un rapatrié. Cette terre est un chez soi, comparée aux pays arabes où les Palestiniens étaient mal traités. La patrie devient le dernier refuge après l'exil dans des pays qui ne respectent pas les droits des réfugiés, disent les personnes interrogées. Beaucoup d'entre eux déclarent que le motif de leur retour est de revenir à la patrie (al watan), même s'ils sont originaires de la Palestine historique.

Le retour des rapatriés n'est pas facile; il s'agit d'une expérience classique de réémigration. Beaucoup rapportent que la population locale ne leur a pas accordé un accueil chaleureux, surtout en Cisjordanie, où ils se sentaient particulièrement peu appréciés. De façon générale, les répondants voient les avantages économiques du rapatriement mais aussi les difficultés d'adaptation au niveau social, pour les rapatriés présents et futurs. Comme le dit un informateur qui travaille dans l'administration de 
l'ANP : «Nous sommes différents (d'eux) au niveau de la culture et du mode de penser.» Paradoxalement la majorité, surtout à Gaza, voit le retour des réfugiés comme un grand avantage, citant par exemple, l'augmentation du nombre de personnes dont le niveau scolaire est élevé et la présence de cultures diverses en Palestine. Certains y voient une revitalisation des valeurs islamiques, ceci probablement à cause des rapatriés venant des États du Golfe. Fait curieux, à Gaza, déjà surpeuplée, la migration de retour est vu comme un moyen d'augmenter la population palestinienne dans les Territoires palestiniens, à la différence de ce que disent les répondants de Cisjordanie.

La vie problématique dans les camps

Plusieurs facteurs peuvent encourager ou non le rapatriement; par exemple, la situation socio-économique, la situation urbaine, le statut des réfugiés palestiniens dans les pays hôtes. Dix ans après Oslo, les camps de réfugiés dans les Territoires palestiniens sont toujours exclus de la planification du développement. La population s'est urbanisée, ce dont témoignent les taux de fécondité qui tendent à rejoindre ceux des villes dans lesquelles ils sont situés. Le taux de fécondité des Palestiniens au Liban est passé de 4,5 enfants en 1987-1990 à 3,9 en 1991-1994, ce qui se rapproche des 2,5 qui constituent la moyenne au Liban, selon les statistiques du Ministère de la Santé en 1995 (Fafo, 2003 : 37).

71 Selon les statistiques de l'UNRWA de 2001, il y a 1460396 réfugiés palestiniens en Cisjordanie et dans la Bande de Gaza, dont 607915 vivant dans les camps: en Cisjordanie ils sont 147884 , soit $24 \%$ des 60777 réfugiés. À Gaza, quelques $53 \%$ des 852626 réfugiés, soit 460031 sont dans cette situation (al-Rimmawi et Bukhari, $2002 \mathrm{p}$. 23-24) (tableau 3).

Tableau 3 : Réfugiés enregistrés par l'UNRWA

\begin{tabular}{|c|c|c|c|c|c|c|}
\hline \multirow[b]{2}{*}{ Région } & \multirow{2}{*}{$\begin{array}{l}\text { Nombre de } \\
\text { camps }\end{array}$} & \multicolumn{3}{|c|}{ Nombre de réfugiés } & \multirow{2}{*}{$\begin{array}{c}\text { \% de réfugiés } \\
\text { vivant dans } \\
\text { les camps }\end{array}$} & \multirow{2}{*}{$\begin{array}{l}\text { Proportion de } \\
\text { réfugiés dans } \\
\text { la population } \\
\text { locale (en \%) }\end{array}$} \\
\hline & & dans les camps & hors des camps & ensemble & & \\
\hline Jordanie & 10 & 287951 & 1351767 & 1639718 & 17,5 & $32,8^{*}$ \\
\hline Cisjordanie & 19 & 170056 & 437714 & 607770 & 27,9 & $31,4^{* * *}$ \\
\hline Gaza & 8 & 460031 & 392595 & 852626 & 53,9 & $78,4^{* *}$ \\
\hline Liban & 12 & 214728 & 168245 & 382973 & 56,0 & $10,7^{*}$ \\
\hline Syrie & 10 & 200054 & 191597 & 391651 & 51,0 & $2,4^{*}$ \\
\hline Totaux & 59 & 1332820 & 2541917 & 3874738 & & \\
\hline
\end{tabular}

L'avenir du rapatriement, tel que nous le présenterons à la fin de cet article, dépendra des améliorations qui seront apportées aux camps dans les Territoires palestiniens, où la population est la plus fragile. Bien que les résidents des camps jouissent de services de santé et d'éducation meilleurs qu'ailleurs, ils sont défavorisés sur le plan économique selon tous les indicateurs ${ }^{12}$, notamment en ce qui concerne le taux de chômage. Par ailleurs, la pauvreté des camps est structurelle : les pauvres en milieu rural peuvent toujours cultiver un lopin de terre pour satisfaire leurs besoins essentiels, tandis que les habitants des camps ne possèdent pas de terre, de façon générale. La fragilité des camps apparait d'autant plus importante quand on compare les employeurs de leurs habitants avec d'autres: $27 \%$ des habitants des camps 
travaillent surtout avec l'ANP, où les salaires sont très modestes, alors que leur proportion est seulement de $19,5 \%$ dans les zones urbaines et $12,8 \%$ dans les zones rurales. En même temps seuls 16,7 \% des résidents des camps travaillent pour les ONG internationales où les salaires sont assez élevés, à rapprocher avec les proportions dans les villes $(15,6 \%)$ et en zone rurale $(26,8 \%)$. Seule exception à cette situation, $5,7 \%$ des employées de l'UNRWA vivent dans les camps, $1,4 \%$ dans les villes, et $0,4 \%$ en milieu rural. Les habitants des camps travaillent moins souvent que les citadins pour le secteur privé ( $34,7 \%$ versus $46,6 \%)$ où les salaires sont moins élevés que dans les ONG, mais un peu plus souvent que les gens de milieu rural $(33,2 \%)$.

La contradiction entre la qualité des services en matière d'éducation et le statut socioéconomique des résidents des camps relève du fait que les personnes dont le statut économique s'améliore tendent à quitter le camp, normalement pour aller dans les grandes villes, où l'offre d'emploi est plus large.

74 La société des Territoires palestiniens n'intègre ni les réfugiés ni les rapatriés, ce pour deux raisons: il s'agit d'une société très fragmentée dont l'intégration dépend de facteurs qui relèvent non seulement du niveau national, mais aussi de la sphère diasporique. Les réfugiés qui vivent ailleurs que dans les camps s'intègrent bien dans les Territoires palestiniens au niveau social et culturel. Pourtant, ceux qui résident dans des camps s'intègrent moins bien aux villes autour d'eux, à en juger par les pratiques matrimoniales. Selon l'enquête PSR de 2003, environ $40 \%$ des réfugiés en dehors des camps ont un membre de la famille qui est marié avec un non réfugié, chiffre qui décroît de moitié pour ceux qui habitent les camps.

La propension à effectuer un retour est liée au caractère de la vie dans les camps. Selon l'étude de Jarrar (2003), les attitudes des réfugiés à l'égard d'eux-mêmes, de la vie des camps et du rapatriement les ont motivés à rentrer, tout en réduisant leurs possibilités d'intégration. Puisque leur estime de soi était élevée mais les conditions de logement étaient difficiles, ils sentaient qu'ils étaient différents des natifs et que leur statut à l'égard des autres était bas. D'autre part, la méfiance et le manque d'harmonie entre eux, comme la réticence des membres des diverses factions politiques à collaborer les uns avec les autres, a réduit leur capacité à obtenir le rapatriement. Les attitudes des réfugiés face à Israël ne sont pas moins contradictoires. D'un côté ils expriment leur amertume, leur peine, et racontent des expériences de mauvais traitements par les Israéliens, ainsi que leur méfiance envers ces derniers. De l'autre, une proportion significative d'entre eux indique qu'ils sont prêts à vivre sous gouvernement israélien et à donner une proportion importante de leurs terres à Israël, si cet État leur permet de revenir. La distinction entre les réfugiés palestiniens qui vivent dans des camps et ceux qui vivent ailleurs est très pertinente en ce qui concerne la propension au rapatriement, comme nous le verrons plus loin.

Le discours nationaliste palestinien du passé revendiquait sa légitimité sur la base de deux points, la Nakba ${ }^{13}$ et le droit au retour des réfugiés. Pour renforcer le nationalisme autant que possible, le camp se présentait comme l'unité primaire de l'identité réfugiée dans les pays hôtes arabes et donc comme le soutien de l'identité palestinienne. En conséquence, le camp devenait une entité quasi-politique, et un objet d'investigation pour une discipline en particulier, les sciences politiques. Les camps se présentaient comme un moyen de reproduction de la société palestinienne de la période d'avant 1948. Dans cette logique, les chercheurs essayaient d'identifier les camps à des lieux d'origine ; par exemple, le camp Al-Huilwa à Lobieh et Yarmouk à Safad... Il y eut ainsi 
une ethnicisation de l'histoire des réfugiés qui a occulté l'importance du rapport économique, social et culturel avec les pays hôtes. Sauf dans un petit nombre d'études sociologiques, anthropologiques, psychologiques et juridiques, les camps ne sont pas abordés en tant que sites urbains.

Des mythes circulaient, non seulement au niveau de l'imaginaire populaire mais aussi dans la communauté scientifique. Par exemple, celui de l'avantage du maintien d'un maximum de Palestiniens dans les camps pour renforcer la mémoire et l'identité palestinienne ou celui selon lequel si les conditions de vie dans le camp restaient misérables, elles limiteraient le nombre de personnes qui allaient vouloir s'établir dans le pays hôte. L'UNRWA et le pays hôte ne peuvent pas exercer leur autorité à moins que les réfugiés ne soient regroupés dans un lieu central et contrôlé où ils sont soumis à une surveillance permanente. Nous pensons qu'il s'agit là d'un discours de stagnation, qui muselle le camp, et que le réfugié peut demeurer tel tout en vivant en dehors du camp.

Les camps dans les Territoires palestiniens sont devenus des symboles de l'illégitimité territoriale pour deux raisons. D'une part, ils sont invisibles par rapport au processus de paix d'Oslo, puisqu'ils sont assimilés aux zones des Territoires palestiniens que l'ANP découpe en espaces de réfugiés et de non réfugiés. D'autre part, les camps se présentent comme des lieux hétérotopiques, au sens de Foucault ${ }^{14}$, déconnectés du tissu social et urbain qui les entoure, espace de tensions autour de la déviation, de la marginalité. La contradiction entre contrôle et mise à la marge favorise des actes potentiels de résistance et transgression. Cette déconnection est le résultat d'un processus graduel qui a été accéléré par l'exclusion des habitants des camps du vote pour les élections locales. Selon Emmanuel Marx (1978), les camps ont perdu leur caractère temporaire et sont devenus des quartiers résidentiels défavorisés.

Cette délégitimation a un impact sur l'identité à plusieurs niveaux. Selon l'enquête Shaml, $89 \%$ des résidents des camps s'identifient à ce lieu et sont fiers d'y habiter. Cependant, certains répondants, surtout dans le camp Shufat, avouent qu'ils cachent leur lieu de résidence à leurs collègues universitaires. Des disputes mineures entre les habitants du camp et ceux de la ville deviennent des conflits majeurs, tels ceux qui se sont produits entre les habitants du camp Kalandia et ceux de Ramallah en 2001. On ne peut comprendre les problèmes des camps de réfugiés que si on les étudie en tant que sites urbains. En raison des longues années de double marginalisation, les camps de réfugiés en Cisjordanie et à Gaza ressemblent à n'importe quel bidonville ou quartier dégradé dans le monde. Les chercheurs doivent les aborder comme tels, comme par exemple ceux de la banlieue parisienne.

Selon l'enquête Shaml, environ les deux tiers des résidents des camps que nous avons interviewés considèrent que leur logement n'est pas assez grand pour leur famille. Environ la moitié juge que les camps ne répondent pas à leurs besoins essentiels ; 57 \% estiment que les conditions sanitaires sont inadéquates. Les deux tiers signalent qu'ils seraient prêts à quitter le camp si leur situation financière s'améliorait.

Enfin, les résidents des camps ressentent leur marginalisation sociale et sont prêts à transformer leur lieu de résidence. Selon l'enquête PSR de 2003, la moitié des réfugiés accepterait de s'installer en dehors du camp, et en accepterait aussi la normalisation, c'est-à-dire, qu'il soit considéré comme partie intégrante de l'agglomération urbaine. Lorsque le camp se situe à l'intérieur de la ville, la majorité de ses habitants (87\%) préfèreraient voter dans le cadre municipal ; la proportion est encore des trois quarts 
quand le camp se situe à l'extérieur. Environ la moitié des résidents est en faveur de l'élargissement du camp à l'intérieur du périmètre urbain.

Évaluation de la propension au retour

82 Le principal résultat de notre recherche concerne la différence considérable entre les Palestiniens qui résident dans les Territoires palestiniens et ceux qui vivent en Israël quant aux divers facteurs qui affecteraient leur mobilité éventuelle. Les Palestiniens des Territoires conservent des liens transnationaux de parenté beaucoup plus denses que ceux qui vivent en Israël. Surtout pour ces derniers, la durée de l'exode, plus de cinquante cinq ans, et la création des frontières ont fait obstacle au maintien des liens de parenté. Le dicton arabe 'al bo'd Jafa' (la distance sèche la relation) n'est pas loin de la vérité. Le système israélien de surveillance et de contrôle des Palestiniens a réussi à créer un décalage énorme entre les deux groupes de Palestiniens.

Le sociologue Aziz Haidar constate que, pendant la période après l'occupation de 1967 où les habitants des Territoires occupés pouvaient encore rendre visite à ceux d'Israël, les rencontres entre les réfugiés palestiniens de Cisjordanie et de Gaza avec leurs parents qui vivaient toujours à l'intérieur des frontières de 1948 étaient assez difficiles ${ }^{15}$. Bien que la première rencontre après de longues années de séparation fût très chaleureuse, les différences ne tardaient pas à se manifester, notamment au niveau social, puisque les Palestiniens qui demeuraient en Israël avaient un niveau de vie meilleur que celui de leurs parents réfugiés qui vivaient en Cisjordanie et à Gaza. Par ailleurs, l'occupation, la surveillance et le contrôle étatiques ont créé un nouveau milieu politique chez les Palestiniens d'Israël. Les visites entre parents ont vite cessé, selon Haidar. Un de nos interviewés, membre du Parti communiste à Haïfa, raconte qu'il trouvait perturbantes ses expériences d'accompagner les Palestiniens venus de l'étranger qui revoyaient leurs maisons, et qu'il a cessé de le faire.

La politique israélienne actuelle relative à l'espace contribue à empêcher les liens entre Palestiniens, même entre ceux qui habitent à l'intérieur de la Cisjordanie et de la bande de Gaza. Les contrôles, qui prennent la forme d'une humiliation, d'une punition collective, créent des frontières dans l'esprit des gens ${ }^{16}$. À cet égard, l'histoire de Barta'a est révélatrice. En 1967, Israël a scindé Barta'a en deux sections, Est et Ouest, de part et d'autre de la Ligne verte. Des familles ont été séparées, de sorte que trente-cinq ans plus tard, elles ne se rencontrent que pour les funérailles et les mariages. On peut imaginer la forte dégradation de la situation avec la construction actuelle du Mur par Israël, tout comme l'évolution des liens entre le Rafah palestinien et le Rafah égyptien, séparés depuis 1983.

Grâce au maintien des liens transnationaux de parenté, le mode familial d'entreprenariat qui prévaut dans les Territoires palestiniens et, dans un moindre degré, en Israël, encouragera beaucoup les exilés qui y participent à retourner vers l'un ou l'autre espace. On peut s'attendre à ce que cette catégorie de migrants fonctionne en mode transnational, en cela qu'ils garderont des liens dans le pays où ils ont vécu ainsi que dans le lieu où ils retournent.

Profil des rapatriés

Bien que le droit au retour ne soit pas le seul facteur qui détermine la migration de retour, il n'en demeure pas moins un élément clef dans le processus puisqu'il élargit les choix possibles pour les réfugiés palestiniens après plus d'un demi-siècle d'exil ${ }^{17}$. L'enquête de terrain menée par Shaml ainsi que les études que nous avons menées dans treize pays de la diaspora palestinienne montrent une population hétérogène de cinq 
millions de personnes, dont seulement une petite partie sera disposée à exercer ce droit. Par exemple, selon l'enquête de Fafo en 2002, un faible pourcentage des Palestiniens du Liban est originaire des Territoires palestiniens ( $1,2 \%$ environ), tandis que la grande majorité provient de la Palestine historique. La situation se complique encore par le fait qu'environ $40 \%$ de la population provienne de localités qui sont devenues complètement juives (par ex. Safad et Tibériade/Bisan) ou bien où les Juifs sont maintenant majoritaires (Acre, Haïfa). Seuls $10 \%$ viennent de villes arabes, comme Nazareth ou le sud palestinien). Bien que deux Palestiniens sur cinq au Liban soient nés en Palestine, très peu ont gardé des liens avec leur terre d'origine (Khawaja, 2003).

Il est difficile d'imaginer un seul scénario pour le retour, en partie à cause des incertitudes qui entourent la question et de la réaction encore inconnue des États $\operatorname{arabes}^{18}$. À la base d'une gamme très large de facteurs qui influenceraient les retours, on peut évoquer trois scénarios possibles qui varient selon deux paramètres: la possibilité d'exercer le droit au retour et le degré d'amélioration des conditions dans les camps de réfugiés. Nos données actuelles ne nous permettent que de proposer des estimations très approximatives du mouvement de retour (tableau 4). Le premier scénario aura lieu dans le cas de l'application du droit au retour sans amélioration dans les camps. Le deuxième concerne l'éventualité où le droit au retour connaît des restrictions imposées par Israël et où la situation dans les camps est améliorée. Le troisième scénario se réalisera si l'application du droit au retour n'est pas garantie.

Tableau 4 : Retour des Palestiniens : trois scénarios

\begin{tabular}{|l|c|c|c|c|c|}
\hline & $\begin{array}{c}\text { Retours des } \\
\text { Territoires } \\
\text { palestiniens } \\
\text { vers Israël }\end{array}$ & $\begin{array}{c}\text { Retours de } \\
\text { réfugiés de } \\
\text { l'étranger } \\
\text { vers Israèl }\end{array}$ & $\begin{array}{c}\text { Retours de } \\
\text { l'étranger } \\
\text { vers les } \\
\text { Territoires } \\
\text { palestiniens }\end{array}$ & $\begin{array}{c}\text { Émigration des } \\
\text { Territoires } \\
\text { palestiniens } \\
\text { vers les pays } \\
\text { occidentaux }\end{array}$ & $\begin{array}{c}\text { Départs des } \\
\text { camps }\end{array}$ \\
\hline Scénario 1 & 200000 & 200000 & 500000 & 47000 & 90000 \\
\hline Scénario 2 & 150000 & 200000 & 500000 & 35000 & 30000 \\
\hline Scénario 3 & 10000 & 20000 & 600000 & 85000 & 30000 \\
& & à 90000 \\
\hline
\end{tabular}

Les rapatriés potentiels ne forment pas un groupe homogène. Au contraire, ils représentent diverses strates sociales, culturelles, politiques et économiques des populations de réfugiés et de la diaspora, qui vont des travailleurs illettrés jusqu'aux entrepreneurs et aux professionnels hautement scolarisées. Il nous est très difficile de prévoir la proportion des diverses catégories socio-économiques parmi les rapatriés, ainsi que le type de retour qui sera réalisé : retour familial définitif, retour individuel, retour provisoire accompagné de comportements transnationaux (maintien de liens entre deux pays, ouverture quant à la possibilité de déplacement, investissement sans retour, séjours courts pour apporter leur expertise dans les Territoires palestiniens...). Une chose est certaine : plusieurs modèles différents émergeront, ce qui sera bénéfique pour l'économie palestinienne, étant donné le mode familial d'entreprenariat et les liens de parenté forts entre la Cisjordanie et la Bande de Gaza et aussi entre les habitants de ces deux espaces et l'étranger, notamment la Jordanie et l'Égypte. L'investissement dans l'économie palestinienne en Israël est moins probable, puisque cette dernière n'est pas autonome par rapport au système israélien; de plus, comme nous l'avons mentionné, les liens des Palestiniens en Israël avec leurs proches à l'étranger sont très faibles. 
89 Il y aura deux mouvements simultanés : celui de la masse des rapatriés et celui d'un groupe plus restreint composé de diplômés et de professionnels, ainsi que d'entrepreneurs. L'élite palestinienne à l'étranger investira son capital et reviendra physiquement, puisqu'elle cherchera à mettre en valeur son statut social dans le pays ou le village d'origine, même quand la situation ne sera pas propice sur le plan de la rationalité économique. Ceci s'observe ailleurs dans plusieurs pays en phase de transition (la Hongrie, la Roumanie, la Bosnie ....) où l'élite de la diaspora joue un rôle politique. Sur la base du taux d'investissement et des activités philanthropiques de la diaspora palestinienne, qu'ils s'adressent à leur famille ou à la société en général, on peut s'attendre à une contribution qui ne serait pas inférieure à un demi milliard de dollars US par année pendant la décennie qui suivra l'indépendance de l'État palestinien.

Conclusion

90 Pour finir, soulignons certains facteurs importants dans l'enjeu. Premièrement, l'importance de l'éducation et du savoir-faire des exilés palestiniens est largement sous-estimée. Plusieurs indicateurs statistiques montrent que le quart des réfugiés dans les pays arabes hôtes sont des entrepreneurs selon la définition de l'OIT (Organisation Internationale du Travail), qu'ils travaillent seuls ou qu'ils aient des employés. Donc, nous nous attendons à ce que le quart des rapatriés puisse bien s'intégrer au marché palestinien. De toute façon, le retour ne sera pas seulement un fardeau pour l'économie palestinienne (ou l'économie arabe en Israël), mais plutôt une force régénératrice, grâce au capital humain et financier que les réfugiés apporteront dans leur pays d'origine. Deuxièmement, la jeune entité palestinienne aura le droit d'adopter une politique sélective face au retour des exilés, comme cela s'est souvent fait ailleurs dans la période qui suit un conflit. Du point de vue historique, la politique de sélection des migrants de retour s'est appliquée dans tous les pays qui ont connu un retour en masse de leurs nationaux, comme ce fut le cas de la migration juive, où la sélection a toujours été fermement contrôlée. Eliezer Kaplan, ministre des finances en 1949 disait, "Nous avons besoin de travailleurs et de guerriers» (cité par Segev, 1986 : 117). Troisièmement, si les États arabes n'obligent pas les réfugiés palestiniens à partir, on peut s'attendre à ce qu'un groupe pionnier d'entre eux revienne au pays, d'où il communiquera un rapport favorable sur la situation à ses réseaux de parents et d'amis, ce qui encouragera ces derniers à revenir à leur tour, et ainsi de suite. Finalement, on peut s'attendre à un retour des retraités, surtout des pays du Golfe et des pays occidentaux, vers les Territoires palestiniens et Israël. Cette forme de «tourisme de longue durée » sera bénéfique à l'économie palestinienne, sans que les migrants aient besoin d'être directement absorbés.

91 Cette recherche met en valeur la complexité d'une migration de retour des Palestiniens. Cette complexité n'est pas fondamentalement différente de celle d'autres expériences de migration de retour ou même d'autres mouvements de réfugiés. Tous ces mouvements migratoires de réfugiés ne se comprennent que dans le cadre plus large de la mondialisation et de la migration internationale. Les migrants internationaux, à savoir ceux qui résident ailleurs que dans leurs pays de naissance, sont estimés à 170 millions d'individus, et ce chiffre ne devrait pas diminuer alors que les pays développés connaissent un déclin et un vieillissement de leur population. Le climat politique de la guerre froide favorisait la réimplantation des réfugiés plutôt que le rapatriement ; aujourd'hui le sentiment anti-immigration défavorise leur installation 
permanente dans le pays de résidence. En dehors de quelques cas d'initiatives bilatérales, c'est le Haut Commissariat aux Réfugiés des Nations unies qui prend en charge l'orientation des cas individuels vers des pays récepteurs (Helton, 2002 : 185).

Les données du HCR de 2002 démontrent que les réfugiés qui exercent leur droit de retour vers leur pays d'origine sont beaucoup moins nombreux (2 252804 , soit $21 \%$ ) que ceux qui choisissent de rester dans le pays hôte ou de s'installer dans un pays tiers. Encore faut-il noter que 2002 fut une année exceptionnelle puisque les Afghans, avec plus de 1800000 individus, représentaient plus que $80 \%$ des migrants de retour. Cet effectif correspond à un peu moins de la moitié de l'ensemble des réfugiés afghans. Les rapatriés provenaient pour la plupart des pays voisins, Pakistan, Iran et Tadjikistan. Ajoutons que les statistiques relatives au mouvement de retour sont souvent trompeuses, puisqu'elles se fondent sur les traversées de la frontière, alors que l'enquête de type anthropologique montre que les réfugiés se sont souvent déplacés à l'intérieur après le retour, comme ce fut le cas des rapatriés de l'OLP vers la Cisjordanie et la Bande de Gaza.

Le faible pourcentage de retour des réfugiés relève de plusieurs facteurs, dont le plus important est la structure du marché du travail mondial. Le cas de la Bosnie est illustratif. Le traité de paix de Dayton à peine conclu, un vif débat a éclaté sur la question du retour. Il s'est intensifié en 1996 quand il est devenu clair que des retours massifs volontaires ne se produiraient pas, du moins pas dans l'immédiat (UNHCR, 2000 : 168). Le débat actuel au sein du HCR tourne autour de cette question: le retour est-il la solution idéale pour les réfugiés ${ }^{19}$ ? Nombre de spécialistes de la migration critiquent la tendance du HCR de favoriser le rapatriement ${ }^{20}$.

L'installation sur le long terme donne lieu à de nouveaux liens entre les réfugiés et le pays hôte. Les paysans s'urbanisent et, dans certains cas, les femmes s'émancipent beaucoup plus que leurs consœurs qui sont restées au pays. Pour ce qui concerne le cas palestinien, il importe de distinguer le droit au retour de la sociologie du retour. Rosemary Sayigh (1977) constate que le retour des Palestiniens dépend non seulement des facteurs «push» qui relèvent du pays hôte mais aussi du désir collectif de retour chez les réfugiés eux-mêmes. Warner, par contre, remet en question la notion du rapatriement, qu'il qualifie d'« idéalisée » et de «nostalgique» (1994:160). Au fil du temps, poursuit l'auteur, la dispersion déforme le sens de la communauté et en même temps, celui de la patrie (voir Zureik, $1997: 80$ ).

\section{BIBLIOGRAPHIE}

ABU-SITTA Salman (2000) Palestinian Right of Return, Sacred, Legal and Possible, London : Palestinian Land Society and Palestinian Return Center, $2^{\text {nd }}$ edition.

AL-RIMMAWI, Hussein and BUKHARI Hana (2002), Population Characteristics of the Population Refugee Camps, Ramallah : PCBS and Dissemination and Analysis of Census Findings (Analytical Report Series (no 3). (In Arabic) 
BASCH L., GLICK SCHILLER Nina and SZANTON BLANC Cristina (1994) Nations Unbound: Transnational Projects, Postcolonial Predicaments, and Deterritorialized Nation-States, New York : Gordon and Breach.

CHARALAMBOS Tsardanidis and HULIARAS Asteris (1999) Prospects for Absorption of Returning refugees in the West Bank and the Gaza Strip, Institute of International Economic Relations, December (Non published report).

ENDRESON Lena and ZUREIK Elia (1996) Studies of Palestinian. An Analytical Bibliography (draft). Presented to the Refugee Working Group Meeting held in Geneva, 12-15 December 1995. Fafo.

ESCWA (1993) Return Migration. Profiles, Impact and Absorption, New York, United Nations, 127 p.

FARGANY Nader (1988) Sa'yan wara' al rizq. Dirasa maydaniyya 'an hijret al masriyyin ll'amal fi al aqtar al arabiyya (Striving for Subsistance), Beyrouth, Centre d'études de l'Unité arabe, $353 \mathrm{p}$.

FOUCAULT Michel (1994) Dits et écrits (IV. 1980-1988), “des espaces autres”, Paris, Gallimard, pp. 752-762.

GEERTZ Clifford (1993) Peddlers and Princes, Chicago : University of Chicago Press.

GRANOVETTER Marc (1985) Economic Action and Social Structure : The Problem of Embeddedness, in American Journal of Sociology, Vol. 91, No 3, November, pp. 481-510.

GRANOVETTER Marc and SWEDBERG Richard (1995) The Sociology of Economic Life, Colorado Westview Press, 391 p.

GRILLO Ralph, RICCIO Bruno and SALIH Ruba (2000) Introduction, in Here or There? Contrasting Experiences of Transnationalism: Moroccans and Senegalese in Italy. Falmer: CDE, University of Sussex.

HANAFI Sari (1996) Between Two Worlds: Palestinian Businessmen in the Diaspora and the Construction of a Palestinian Entity, Cairo, CEDEJ for the French edition and two editions in Arabic : Cairo, Dar alMostaqbal al-arabi, \& Ramallah, Muwatin, (in Arabic).

HANAFI Sari (1997) Entre deux mondes. Les hommes d'affaires palestiniens de la diaspora et la construction de l'entité palestinienne, Le Caire, CEDEJ, 1997.

HANAFI Sari (1998a) Business Directory of Palestinian in the Diaspora, Jerusalem: Biladi, June 1998 (en anglais, français et arabe).

HANAFI Sari (1998b) "Contribution de la diaspora palestinienne à l'économie des Territoires : investissement et philanthropie", in Maghreb-Machrek, n 161 novembre 1998, pp. 59-68.

HANAFI Sari (1999) "Investment by the Palestinian Diaspora in the manufacturing Sectors of the West Bank and Gaza Strip", in ESCWA (ed.), Proceedings of the Expert Group Meeting on the Impact of the Peace Process on Selected Sectors, Amman : USCWA, p. 201-26.

HANAFI Sari (2001) Here and There: Towards an Analysis of the Relationship between the Palestinian Diaspora and the Center, Ramallah : Muwatin, Jerusalem: Institute of Jerusalem Studies (distribution Beirut : Institute of Palestine Studies) (in Arabic)

HANAFI Sari (2001b) "La Diaspora palestinienne et la conversion des capitaux issus de la rente pétrolière", Tiers Monde, Paris, $\mathrm{n}^{\circ}$ 163, pp. 623-643.

HANAFI Sari (2002) Opening the Debate on the Right of Return, MERIP, Washington, March.

HANAFI Sari (Forthcoming) "Rethinking the Palestinians Abroad as a Diaspora : The Relationships between the Diaspora and the Palestinian Territories", HAGAR. International Social Science Review, Vol. 4 (1-2), 2003, pp. 157-182. 
HARVEY David (1990) The Condition of Postmodernity, Blackwell.

HEIBERG Marianne and OVENSEN Geir, 1993, Palestinian Society in Gaza, West Bank and Arab Jerusalem : A survey of Living Conditions, Fafo-report 151, Oslo.

HEACOCK, Roger (1999) The Becoming of Returnee States: Palestine, Armenia, Bosnia, Beirzeit: Beirzeit University.

HOLY Ladislav (1989) Kinship, honour and solidarity: cousin marriage in the Middle East. Manchester: Manchester University Press.

ILO (1998) Resolution concerning the Measurement of Underemployment and inadequate employment situations, The Sixteenth International Conference of Labour Statistics, Geneva, ILO.

ISOTALO Riina (1997) Yesterday's Outsiders, Today's Returnees - Transnational Processes and Cultural Encounters in the West Bank, in A. Linjakumpu and K. Virtanen (eds) Under the Olive Tree. Reconsidering Mediterranean Politics and Culture. Tampere: European Science Foundation and Tampere Peace Research Institute.

ISOTALO Riina (2002) Gendering the Palestinian Return Migration: Migrants from the Gulf and Marriage as a Transnational Practice, Paper presented at the Third Mediterranean Social and Political Research Meeting, Florence, March 20-24, 2002, Mediterranean Program, Robert Schumann Centre for Advanced Studies, European University Institute.

JABER Hanna (1997) Le camp de Wihdat à la croisée des territoires, in Riccardo Bocco, Blandine Destremau et Jean Honnoyer (eds.), Palestine, palestiniens. Territoire national, espaces communautaires, Amman, CERMOC.

JACONSEN Laurie Blome and DEEB Mary (2003) Social Network, in Ugland, Ole Fr. (ed.) Difficult Past, Uncertain Future. Living Conditions Among Palestinian Refugees in Camps and Gatherings in Lebanon, Fafo Report 409 (www.fafo.no)

JARRAR, Najeh (2003) Palestinian Refugee Camps in the West Bank: Attitudes Towards Repatriation and Integration, Ramallah: Palestinian Diaspora and Refugee Center, Shaml.

KHAWAJA Marwan (2003) Population, in Ugland Ole Fr. (ed.), Difficult Past, Uncertain Future. Living Conditions Among Palestinian Refugees in Camps and Gatherings in Lebanon, Fafo Report 409 (www.fafo.no)

KHAWAJA Marwan and TILTNES Åge A. (eds.) (2002) On the Margins: Migration and Living Conditions of Palestinian Camp Refugees in Jordan. Norway: Fafo.

KING R.E. (1984) Return Migration and Regional Economic Problems, London, Croom Helm.

KIRZNER Israel (1982) "Uncertainty, Discovery, and Human Action: A Study of the Entrepreneurial Profile in the Misesian System", in Kirzner, Israel ed., Method, Process, and Austrain Economics: Essays in Honour of Ludwig Won Mises, Lexington Books, Lexington (Mass.), pp. 139-159.

MALKI Majdi and SHALABI Yasser (2000) Internal Migration and Palestinian Returnees in West Bank and Gaza Strip. Ramallah : MAS (en arabe avec résumé en anglais)

MASSEY W. et al. (1993) "Networks of infinite-server queues with nonstationary Poisson input". Queueing Systems Theory Appl. 13 (1993), no. 1-3, 183-250.

MELTON Arthur (2002) The Price of indifference. Refugees and Humanitarian Action in the New Century, Oxford University Press. 
MOPIC (Ministry of Planning and International Cooperation) (1998) "Aid Coordination Department. MOPIC's 1997 Fourth Quarterly Monitoring Report of Donor Assistance", in Web Site www.pna.net, Ramallah.

ONG A. (1999) Flexible Citizenship. The Cultural Logic of Transnationality. Durham and London: Duke University Press.

PEDERSEN Jon et al. (ed.) (2001) Growing Fast. The Palestinian Population in the West Bank and Gaza Strip, Norway, Fafo, 194 pages.

POLANYI Karl (1957) The Economy as Instituted Process, in The Great Transformation; reprint, Boston, Beacon Press.

PORTES Alejandro 1998, Social Capital: Its Origins and Applications in Contemporary Sociology, Annual Review of Sociology 24, pp. 1-24.

PUTNAM, R. D. (2000) Bowling Alone. The collapse and revival of American community, New York: Simon and Schuster

REYNOLDS Paul D., HAY Michael and CAMP S. Michael (1999) Global Entrepreneurship Monitor, 1999 Executive Report, Babson College/Kauffman Center for Entrepreneurial Leadership/London Business School.

ROY Sara (1995) The Gaza Strip: The Political Economy of De-development, Washington, Palestine Studies Institute.

SAAD al Din et FADIL Abdel (1983) Intiqal al 'amalah al 'arabiyya: (The Movement of Arab Labor). Beirut: Center of Arab Unity Studies.

SALIH Ruba (2000) "Transnational Practices and Normative Constraints Between Morocco and Italy : A Gendered Approach", unpublished paper presented at the First Mediterranean Social and Political Research Meeting, March 22-26, the Mediterranean Programme of the Robert Schumann Centre at the European University Institute in Florence, Italy.

SIMEON Richard (1997) Citizen and Democracy in the Emerging Global Order, in Thomas J. Coutchen (ed.) The Nation State in a Global/Information Era : Policy Challenge, Canada : Johen Deutsch Institute for the Study of Economic Policy.

SLETTEN Pal and PEDERSEN Jon (2003) Coping with Conflict. Palestinian communities Two Years into the Intifada, Norway : Fafo (also www.fafo.no)

SMITH R. (1998) Reflections on Migration, the State and the Construction, Durability and Newness of Transnational Life in Soziale Welt Transationale Migration, 12. Baden-Baden, Nomos Verlagsgesellschaft.

SAYIGH Rosemary (1977) "Sources of Palestinian Nationalism: A Study of a Palestinian Camp in Lebanon", Journal of Palestinian Studies, vol. 6, no 4.

SEGEV Tom (1949) The First Israelis, Free Press, 1986

TIMMONS Jeffry A., SMOLLEN Leonard E. and DINGEE Alexander L.M. Jr. (1990) New Venture Creation: Entrepreneurship in the 1990s, Homewood/Boston : Irwin.

TUASTAD D. (1994) "Finding Ways. Palestinian Coping Strategies in Changing Environments" in Gilen S.; Hovdenak A.; Maktabi R.; Pedersen J.; Tuastad D. ed.: Fafo Report no 34. Fafo, Oslo.

UNHCR (2000) Les réfugiés dans le monde. Cinquante ans d'action humanitaire, Paris, Autrement, 338 p. VAN HEAR Nicholas (1997) Reintegration of the Palestinian Returnees, Monograph No 6, Ramallah, Shaml Publications. 
WARNER Daniel (1994) Voluntary Repatriation and the Meaning of Returning Home: A Critique of Liberal Mathematics. Journal of Refugee Studies 7 (2/3), pp. 160-174.

ZUREIK Elia (1997) The Trek Back Home : Palestinians Returning Home and their Problem of Adaptation in Hovdenak Are and al. Constructing Order : Palestinian Adaptation to Refugee Life, Jerusalem, Fafo, Institute for Applied Social Science.

\section{NOTES}

1. -Le concept de capital social a acquis une prééminence remarquable dans la littérature théorique et appliquée au cours de la dernière décennie. Un vif débat a accompagné cette montée, ce qui a fait progresser la réflexion sur le sujet, tout en clarifiant les zones d'accord et de désaccord ; cependant, beaucoup reste à faire. Notre usage du terme « capital social » diffère de celui de Robert Putnam (2000). Dans cette étude nous nous intéressons principalement à la contribution de la famille et au degré de pertinence de l'entreprenariat familial et ethnique.

2. - Cette enquête a été conduite par le Palestinian Diaspora and Refugee Center, Shaml de janvier à octobre 2000 Elle a bénéficié d'un financement du Centre International de Recherche et Développement (Ottawa). L'auteur remercie tous les participants à la recherche, en particulier Shirine Araj. Il remercie aussi ceux qui ont enrichi l'enquête par la discussion : Roula El-Rifai, Pamela Scholey, Riina Isotalo et Cedric Parizot. Au total 560 questionnaires ont été renseignés, non seulement par des réfugiés mais aussi par d'autres Palestiniens habitant les Territoires palestiniens ou Israël. L'enquête en Israël a été réalisée en collaboration avec le Centre Mada à Haifa. D'autres enquêtes telles celle sur les conditions de vie des réfugiés palestiniens produites par Fafo en Jordanie et au Liban aussi bien que le Palestinian Center for Policy and Survey Research (PSR) seront utilisés à fins de comparaison. L'enquête de Fafo concerne un échantillon de 4000 familles distribuées dans 12 camps en Jordanie alors que l'enquête au Liban concerne 3100 familles avec une étude approfondie fondée sur les discussions avec 13 focus groups. L'enquête de PSR est concentrée surtout sur les réfugiés palestiniens et leurs problèmes. Elle a été réalisée en janvier 2003 et a visé 1498 ménages de réfugiés palestiniens situés dans 150 localités en Cisjordanie et dans la bande de Gaza.

3. -On peut critiquer nombre d'enquêtes réalisées dans les Territoires palestiniens en raison de la teneur émotive des questions posées.

4. -L'Institut de Santé Publique de Birzeit étudie cette question actuellement.

5. -Son étude porte sur deux camps de réfugiés, Fari'a et Balata. Les données ont été recueillies au moyen de deux méthodes, le questionnaire et l'entretien approfondi. Le premier a été appliqué à un échantillon aléatoire de $5 \%$ du total des ménages dans les deux camps, soit 58 des 374 de celui de Fari'a et 86 des 801 de celui de Balat. Cet échantillon a été extrait d'une liste d'unités domestiques fournie par le bureau du Directeur de l'UNRWA dans chaque camp.

6. - Cette catégorie inclut les tantes et oncles matrilatéraux et patrilatéraux et leurs familles.

7. -Tous les pourcentages se réfèrent à l'enquête de Shaml sauf mentionne contraire.

8. -Avantageux, parce que le prix de la fiancée demandé à la famille du mari tend à être moindre dans ce cas, ce qui a pour effet de préserver les ressources familiales.

9. $-82 \%$ des répondants affirment que la situation était la même avant l'intifada. 
10. -Ce modèle se retrouve dans d'autres groupes diasporiques (Ong, 1999:20) où les normes culturelles entourent la formation de réseaux transnationaux commerciaux. Chez les Chinois d'outremer, les hommes sont mobiles tandis que les femmes et les enfants restent soumis au joug des régimes familiaux.

11. Certains dans ce groupe étaient admis au pays dans le cadre de la réunification familiale ou avec un visa temporaire de visite. Ces derniers sont restés dans les Territoires palestiniens bien que leur statut soit irrégulier selon les règlements israéliens.

12. - Toutes les statistiques qui suivent sont extraites du recensement de 1997 (al Rimmawi et Bukhari, 2002). Il existe des chiffres plus récents mais nous avons préféré utiliser ceux de 1997, pour neutraliser l'effet de l'Intifada et ainsi mieux apercevoir la situation de l'avenir.

13. - Catastrophe. Ce terme désigne l'expulsion massive de Palestiniens en 1947-1949.

14. -Par opposition aux utopies, lieux irréels, Foucault qualifie d'hétérotopies ces lieux réels et effectifs, qui sont « des sortes de contre-emplacements réels, sortes d'utopies effectivement réalisées, (...) des sortes de lieux qui sont hors de tous les lieux, bien que pourtant ils soient effectivement localisables. » (Foucault, 1994 : 755).

15. - Communication personnelle.

16. -Traverser le point de contrôle le plus important de Cisjordanie est permis seulement à ceux qui peuvent démontrer qu'ils sont domiciliés de l'autre côté. Tout autre motif - visites, école, travail, maladie, affaires, achats - conduit à un refus. Pendant la fête la plus importante du calendrier musulman en 2002, les Palestiniens ne pouvaient se déplacer entre Ramallah et Bethlehem. "Ce n'était pas vraiment une fête à ce que nous pouvions voir, sauf pour l'habillement des gens et les cadeaux qu'ils portaient. Tout un peuple qui voyage à pied sur le terrain rocailleux, leurs paquets et cadeaux à la main, le désespoir au coeur. Le cœur même de leur terre est parsemé de points de contrôle, et à chacun la violence menace » (Levy, 2002).

17. -Par ailleurs, nous pensons que le droit au retour est aussi la clef de toute solution durable au conflit entre Palestine et Israël, puisque la fin de l'occupation des terres palestiniennes requiert que les Israéliens réfléchissent sur leur responsabilité morale quant à la genèse du problème des réfugiés palestiniens et sur le conflit de 1948.

18. -Un Palestinien qui vit au Liban risque d'avoir du mal à décider de revenir si la position du Liban n'est pas claire. Est-ce que les Palestiniens seront rejetés à la frontière comme ce fut le cas en Libye au début des années 1990 ou auront-ils le droit de choisir?

19. -Le retour forcé de réfugiés au Rwanda en 1996 a suscité bien des critiques. Le HCR a été accusé pour avoir permis, en violation de la Convention de Genève, le renvoi de réfugiés là où ils pouvaient être persécutés (Melton, 2002, p. 22).

20. -Entrevue avec Barbara Harell-Bond et Fabienne Le Houérou au Caire, avril 2003. 


\section{RÉSUMÉS}

Cet article cherche à identifier les modèles de retour et de transnationalité. Il met en relief les liens de parenté sociale et économique entre les Palestiniens à l'intérieur et à l'extérieur des Territoires Palestiniens, ainsi que le mode d'entreprenariat qui s'y développe. À la différence d'autres études qui portent sur l'absorption de réfugiés, centrées uniquement sur la contribution de l'État et de la communauté internationale pour faciliter le retour, nous nous intéressons à l'examen du capital social, qui peut encourager le retour ou l'adaptation au nouvel environnement. Ce texte sera centré sur les facteurs objectifs qui influencent la décision du retour, tels que les réseaux sociaux et économiques de la parenté, l'entreprenariat et la culture migratoire, et non sur les attitudes fondées sur les émotions, qui expriment la position politique du sujet mais pas nécessairement son intention concernant le retour. Quel type de retour aura lieu? Quel sera le profil des rapatriés ? Y aura-t-il des masses de réfugiés qui se précipiteront en même temps, ou plutôt des filets de groupes fragmentés ? Quelle sera la motivation du retour : le nationalisme et le désir de stabiliser l'identité (ou les identités) après l'expérience de l'exil, ou plutôt quelque chose qui les dépasse ? Dans le cas où Israël accepte le principe du droit au retour, ce dernier sera-t-il volontaire ou forcé ? Qu'est-ce qui constitue, au fait, le retour " dans la sécurité et la dignité " ? Les réfugiés devraient-ils être obligés de revenir s'ils ne peuvent pas retourner dans leurs localités d'origine, mais ailleurs dans le pays? Voilà plusieurs questions auxquelles cette étude vise à répondre.

The impact of Social Capital on the Eventual Repatriation Process of Refugees. Preliminary Study of Economic and Social Transnational Kinship Networks in Palestine/Israel.--This article identifies patterns of return and transnationalism that may already be observed. The analysis highlights the role of kinship networks and economic ties linking Palestinians inside and outside the Palestinian territories. Unlike other studies on the absorption of refugees, which focus only on the contribution of the state and the international community in facilitating the return, I am more interested examining sociological factors that can encourage return and/or adaptation to the new economic environment. Therefore, I focus on the objective factors that influence the decision for return migration, such as economic and social kinship networks, entrepreneurship and migration culture, as opposed to emotionally-based attitudes, which express the subject's political position but not necessarily their actual intention to return. What pattern of return will take place and what will be the profile of the returnees? Will there be a mass of refugees rushing in simultaneously or a trickle of fragmented groups? What is the motivation for return: pure nationalism and the desire to stabilize identity(ies) after the experience of exile, or something extending beyond that? If Israel accepts the principle of the right of return, would return be voluntary or coerced? What constitutes return 'in safety and dignity'? Should refugees be required to return if they cannot go back to their areas of origin, but must settle in another part of the country? These are some of the questions that this chapter attempts to address.

El impacto del capital social en el proceso de la repatriación de los refugiados. Un estudio preliminar de las redes económicas y sociales de parentesco en Palestina e Israel. -- Este artículo trata de identificar los modelos de retorno y de transnacionalidad. Son subrayados los lazos de parentesco social y económico entre los palestinos del interior y del exterior de los Territorios Palestinos, así como el modo empresarial que dichos lazos desarrollan. Al contrario de otros estudios relativos a la absorción de los refugiados, únicamente centrados en la contribución del Estado y de la comunidad internacional para facilitar su retorno, este artículo examina el capital social que puede animar a la vuelta o bien a la adaptación al nuevo ambiente. Este texto se centra en los factores objetivos que influencian la decisión del retorno tales como las redes sociales y 
económicas del parentesco o la empresa y la cultura migratoria. Este estudio descarta pues las actitudes de tipo emocional que expresan la posición política del sujeto mas no necesariamente su intención de volver. ¿Qué tipo de retorno tendrá lugar? ¿Cuál será el perfil de los repatriados? ¿Habrá masas de refugiados que se precipitarán al unísono o más bien una corriente de grupos fragmentados? ¿Cuál será la motivación del retorno: el nacionalismo y el deseo de estabilizar la identidad (o las identidades) tras la experiencia del exilio, o algo que les supera? En caso de que Israel acepte el principio del derecho al retorno, ¿será éste voluntario o forzoso? ¿Qué es lo que constituye, de hecho, el regreso "seguro y digno"? ¿Deben ser obligados los refugiados a volver si no pueden hacerlo a sus localidades de origen? Estas son algunas de las cuestiones a las que este estudio pretende responder.

\section{INDEX}

Mots-clés : parenté, rapatriement, réfugiés, réseaux sociaux

Index géographique : Israël, Palestine

\section{AUTEUR}

\section{SARI HANAFI}

Directeur du Palestinian Diaspora and Refugee Center, Shaml (Ramallah). hanafi@p-ol.com 